Check for updates

Cite this: RSC Adv., 2017, 7, 26735

\title{
Macroscopic assembly of flexible and strong green graphene fibres $\uparrow$
}

\author{
R. Bakhtiari, ${ }^{a}$ S. Ghobadi, ${ }^{a}$ E. N. Güllüoğlu, ${ }^{\text {b }}$ L. I. Şanlı, ${ }^{c}$ S. A. Gürsel ${ }^{\text {ac }}$ and E. Özden- \\ Yenigün (D)*bd
}

\begin{abstract}
Graphene fibres have great potential in future wearable electronics due to their promising thermal and electrical properties. However, fibre brittleness limits their implementation and researchers are still seeking easily scalable and eco-friendly production methods. Here we propose a green and continuous wet-spinning assembly approach to continuously spin flexible graphene oxide (GO) fibres. Highly stable aqueous GO suspensions up to $40 \mathrm{mg} \mathrm{mL}^{-1}$ are achieved and GO fibres are spun from highly oriented liquid crystals through a customized continuous fibre production line. As-spun GO fibres with specific ultimate tensile strength of $7 \mathrm{~N} /$ tex show strain to failure (\%) of $10 \%$; subsequent $\mathrm{NaBH}_{4}$ chemical reduction gives graphene fibres with electrical conductivity of $214 \mathrm{~S} \mathrm{~cm}^{-1}$. The scalable production presented here facilitates flexible, strong and electrically conductive graphene fibres, which have emerged as promising graphene based electronic textiles and sensors.
\end{abstract}

Received 7th April 2017

Accepted 15th May 2017

DOI: 10.1039/c7ra03975a

rsc.li/rsc-advances

mechanical, electrical and morphological properties. ${ }^{15}$ The

\section{Introduction}

As a well-known two dimensional (2D) natural material, there have been numerous reports of graphene's superior electrical and mechanical characteristics, compared to other carbon nanomaterials. ${ }^{1,2}$ Graphene's properties, empowered by its unique $\mathrm{sp}^{2}$ formation of carbon atoms leading to $2 \mathrm{D}$ layered structures, are desired in a variety of disciplines. ${ }^{3,4}$ These superior properties make graphene a perfect candidate in various fields including composites as reinforcement additives, ${ }^{5,6}$ clean and renewable energy production ${ }^{1-4}$ biocompatible thin films, ${ }^{1-7}$ and nanoelectronics. ${ }^{8}$ There have been several reports of different forms of graphene-based substances. ${ }^{5,9}$ Among which, the three-dimensional (3D) graphene fibres exhibit promising electrical, mechanical and thermal properties in macroscopic scale. ${ }^{10}$ The previous studies have led to formation of graphene oxide (GO) aqueous liquid crystals (GOLCs) for fibre spinning.,911-14 The key of GOLCs preparation is to achieve the uniaxial alignment of GO layers called a nematic phase, ${ }^{12}$ which is required for the optimum

${ }^{a}$ Faculty of Engineering and Natural Sciences, Sabanci University, 34956 Istanbul, Turkey

${ }^{b}$ Istanbul Technical University, Faculty of Textile Technologies and Design, Department of Textile Engineering, 34437, Istanbul, Turkey. E-mail: ozdenyenigun@ itu.edu.tr

'Nanotechnology Research and Application Center (SUNUM), Sabanci University, 34956 Istanbul, Turkey

${ }^{d} I T U$ Aerospace Research Center, Istanbul Technical University, Istanbul, 34469, Turkey

$\dagger$ Electronic supplementary information (ESI) available. See DOI: 10.1039/c7ra03975a substantial increase in alignment of GOLCs leads to better 3D macroscopic fibres as demanded both in academia and industry, ${ }^{911-14,16,17}$ due to their high specific surface area and high tensile strength compared to other particle geometries. ${ }^{2,18,19}$ The wet-spinning method, as a well-known approach for preparation of polyacrylonitrile based carbon fibres, possesses several advantages including easy scalability, low cost of operation, higher processing yield and eco-friendly manufacturing. ${ }^{20}$ There are several studies about the fabrication of graphene fibres however, to the best of our knowledge a very few of them offer continuous flexible green fibre production with the potential of scalability. For instance, Gao et al., ${ }^{11}$ prepared graphene fibres using wet-spinning method and the concentration of graphitic nanosheets used in the GOLCs was lower than $10 \mathrm{mg} \mathrm{mL} \mathrm{m}^{-1}$ whereas hydroiodic acid (HI) chemical reduction which prompts release of caustic byproduct gases, was used. Besides, the low density of GO nanosheets in GOLCs and harsh reduction methods resulted in low layer packing inside the fibres, thus reduced their mechanical properties. In another study, Yu et al. ${ }^{21}$ produced pristine graphene fibres by a similar approach using surfactant coagulation bath. They observed better alignment of GO nanosheets in the GOLCs which leaded to higher packing density of graphene layers and better resultant properties. Not only GOLCs characteristics but also the selection of coagulation media is an essential parameter which affects fibre tensile properties. As shown in Table 1, the GO fibres tensile strength coagulated in $\mathrm{CaCl}_{2}$ bath is higher than those prepared in different coagulation media such as $\mathrm{CTAB}$, ethanol and $\mathrm{NaOH}^{13,21,22}$ In wet spinning of GO fibres, the elimination of functional groups 
Table 1 Summarizes the studies conducted in literature by revealing process parameters and their final properties (hydroiodic acid (HI), cetyl trimethylammonium bromide (CTAB) and deionized water (DI), potassium hydroxide $(\mathrm{KOH})$, copper(I) sulfate $\left.\left(\mathrm{CuSO}_{4}\right)\right)$

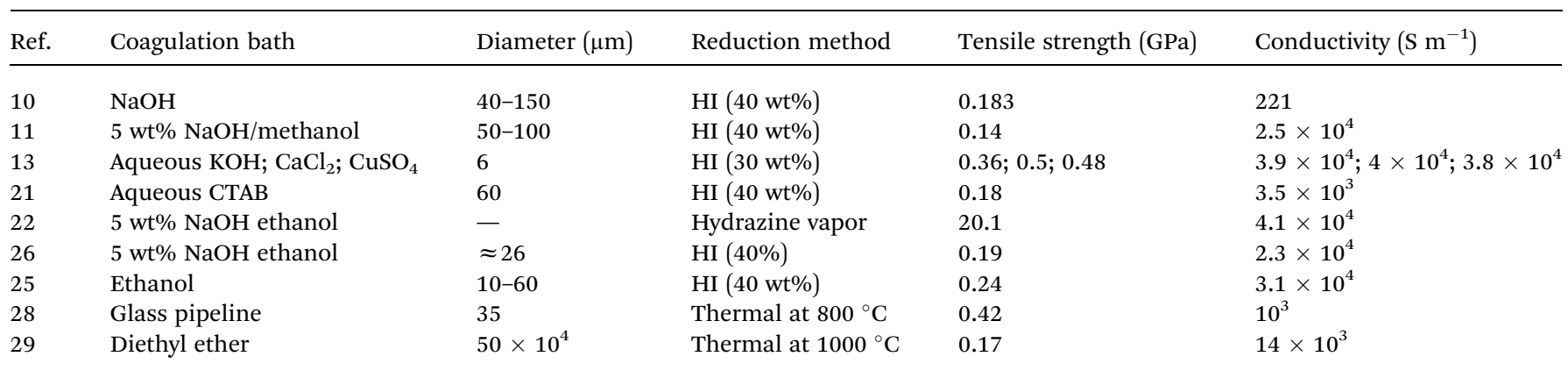

present on the graphene layers are required to conduct electrons. Among different approaches such as thermal reduction, ${ }^{5}$ microwave irradiation, ${ }^{23}$ chemical reduction ${ }^{24}$ method was the most reliable one without sacrificing the mechanical performance. At the same time, the defect density of graphene nanosheets is also tuneable compared to thermal reduction approach..$^{11,13,25-27}$

In this study, we aimed to propose scalable and green production of GO and graphene fibres without sacrificing mechanical and electrical properties. First, a mild process of GO synthesis based on Hummer's modified method was conducted while the temperature was reduced and via substitution of nitric acid with phosphoric acid production of $\mathrm{NO}_{x}$ pollutant gasses were eliminated. ${ }^{24,30}$ Afterwards, the GOLCs with dominant nematic phase formation were prepared via ultrasonic homogenization of GO nanosheets in aqueous media. At this stage, no surfactant and/ or stabilizing agent was used to achieve $40 \mathrm{mg} \mathrm{mL}^{-1} \mathrm{GO}$ concentration with densely packed GO nanosheets. Thus, pristine GO fibres were produced by avoiding the inevitable effect of surfactants and stabilizers on mechanism of electrical conduction. Then, GOLCs were wet-spun and for the first time systematically optimized fibre production line was designed for continuous, strong and electrically conducting graphene fibres. Various processing conditions (e.g. the feeding rate, take up speed and drawing speed) and coagulation composition were investigated and optimized through production (Fig. 1). For fibre finishing, another systematic study on the improvement of electrical conductivity was conducted by exploring the effect of different chemical reduction methods. To the best of our knowledge, this study reports highest specific tensile strength for flexible and electrically conductive graphenebased single fibres, and also reveals the prerequisites of better mechanical, electrical and morphological properties by discussing the optimal material and process parameters. We should also emphasize that starting from GO synthesis to GO fibre reduction, green strategies including material and method selection has been promoted for flexible graphene fibre production.

\section{Experimental section}

\section{Graphene oxide (GO) synthesis via chemical oxidation}

Graphite flakes (particle size +100 mesh ( $\geq 75 \%$ min) and melting point $\left.3652-3697^{\circ} \mathrm{C}\right)$, potassium permanganate $\left(\mathrm{KMnO}_{4}\right)$, sulfuric acid $\left(\mathrm{H}_{2} \mathrm{SO}_{4}\right)$, phosphoric acid $\left(\mathrm{H}_{3} \mathrm{PO}_{4}\right)$, hydrogen peroxide $\left(\mathrm{H}_{2} \mathrm{O}_{2}\right)$, hydrogen chloride ( $\mathrm{HCl})$, sodium borohydride $\left(\mathrm{NaBH}_{4}\right)$, hydroiodic acid (HI), ascorbic acid (AA), calcium chloride $\left(\mathrm{CaCl}_{2}\right)$, sodium hydroxide $(\mathrm{NaOH})$, and ethanol were purchased from Sigma-Aldrich and used without further purification. The improved synthesis protocol was tuned according to Hummer's method during which, $\mathrm{KMnO}_{4}$ was mixed mechanically with natural graphite flakes. As a substantial change of nitric acid $\left(\mathrm{HNO}_{3}\right)$ in Hummers' modified method, ${ }^{24} \mathrm{H}_{3} \mathrm{PO}_{4}$ was used in this study to have mild conditions ${ }^{30}$ using the same protocol. ${ }^{5}$ First, one equivalent mass of graphite powder was put into glass flask placed in oil bath followed by pouring and mixing of $\mathrm{KMnO}_{4}$ and graphite flakes with $6: 1$ mass ratio, respectively. Then, with respect to graphite flake mass fraction, $9: 1$ and $1: 1$ mass ratios of $\mathrm{H}_{2} \mathrm{SO}_{4}$ and $\mathrm{H}_{3} \mathrm{PO}_{4}$, was added slowly to the initial solid mixture and distilled. The mixture was kept for 24 hours and then cooled by using ice bath. The resultant brownish slurry was introduced to the $20: 1(\mathrm{v} / \mathrm{v})$ ice $-\mathrm{H}_{2} \mathrm{O}_{2}$ mixture for further neutralization. The final GO powder was washed with DI water and ethanol aqueous solutions several times. The GO nanosheets were dried for 48 hours at $70{ }^{\circ} \mathrm{C}$ and conditioned.

\section{GOLCs formation via high energy sonication}

The chemical functionalization provides controlled exfoliation of graphite layers with hydrophilic functional groups. ${ }^{5}$ Our functionalized GO can be readily dispersed in hydrous media with mild ultrasonic treatment due to the hydrogen bonding affinity of the existed surface functional groups. GOLCs were prepared in DI water at four different GO concentrations as 20, 25, 30 and $40 \mathrm{mg} \mathrm{mL}{ }^{-1}$. Each sample was sonicated during different consecutive periods (4 hours with cool-down intervals) in sonication bath (power: 340 watts) and later high power probe sonicated (power: 650 watts) as detailed in Table S1. $\dagger$ At higher GO concentrations slightly higher energy was consumed. By eye examination, there is no changes observed at each GO concentration level due to tuned ultrasonication power.

\section{Wet-spinning of GO fibres}

Meter-scale continuous graphene fibres were produced by customdesigned wet-spinning line (Fig. 1). For continuous production of neat GO fibres, the condensed aqueous GOLCs were injected through spinneret into the coagulation media at a rate of $60 \mu \mathrm{L}$ $\min ^{-1}$. Based on previous studies, several chemicals (e.g. KOH, 


\section{GO synthesis via chemical oxidation}
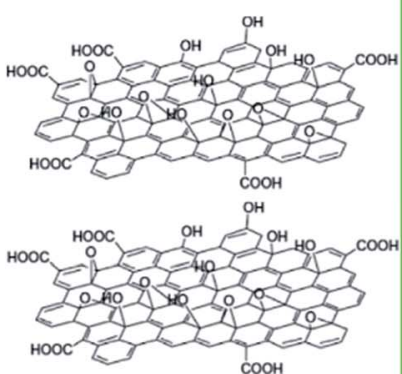

$\mathrm{COOH}$

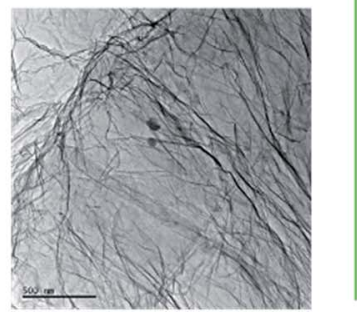

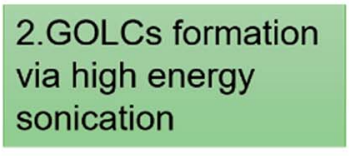
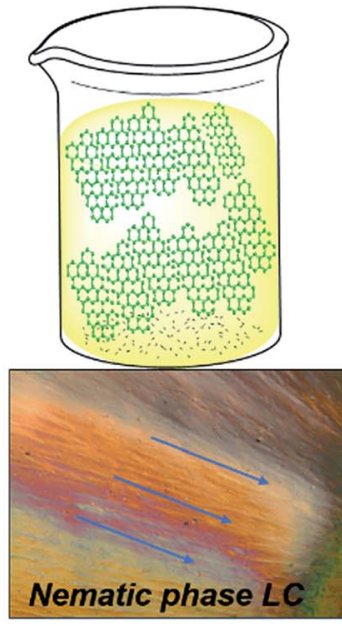
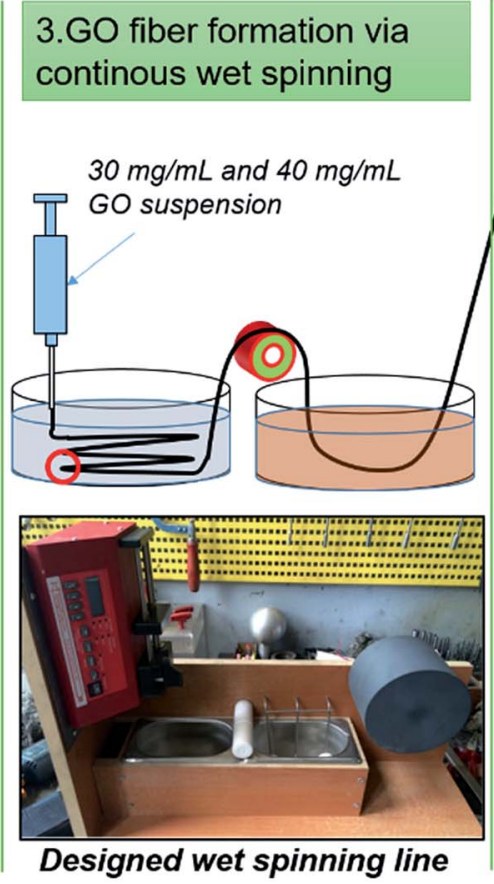

4.Continous GO fiber formation
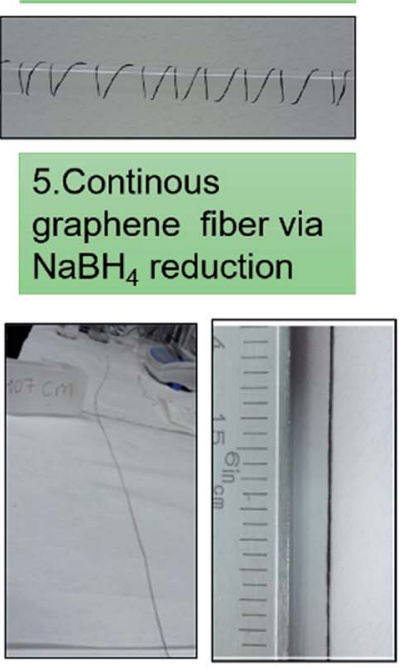

Fig. 1 Schematic of continuous GO and electrically conducting graphene fibre formation via designed wet-spinning production line.

$\mathrm{CuSO}_{4}, \mathrm{NaOH}, \mathrm{CaCl}_{2}$ ) were used for coagulation, however the best reported results were obtained at the coagulation baths of $5 \mathrm{wt} \%$ and $3 \mathrm{wt} \% \mathrm{NaOH}$ in ethanol and $\mathrm{CaCl}_{2}$ in $3: 1(\mathrm{v} / \mathrm{v})$ ethanol : DI water solutions. The solidified GO fibre was collected onto the spinning drum at $70 \mathrm{rpm}$. The process was followed by washing with methanol to remove the residual coagulation chemicals. The collected fibres were dried for 24 hours at room temperature and conditioned. The wet-spinning process was conducted by the continuous spinning/washing custom setup (Fig. 1) and Table 2 gives the detailed fabrication parameters and the sample coding of

Table 2 GO concentration, coagulation bath composition and sample coding of as-spun GO fibre

\begin{tabular}{|c|c|c|c|}
\hline GO fibre & $\begin{array}{l}\mathrm{GO} \\
\text { concentration } \\
\left(\mathrm{mg} \mathrm{mL}^{-1}\right)\end{array}$ & $\begin{array}{l}\text { Coagulation bath } \\
\text { concentration } \\
(\mathrm{wt} \%)\end{array}$ & $\begin{array}{l}\text { Coagulation bath } \\
\text { composition }\end{array}$ \\
\hline G20N3 & 20 & $\mathrm{NaOH}(3)$ & Ethanol \\
\hline G20N5 & 20 & $\mathrm{NaOH}(5)$ & Ethanol \\
\hline G20C3 & 20 & $\mathrm{CaCl}_{2}(3)$ & Ethanol : water $(3: 1)$ \\
\hline G20C5 & 20 & $\mathrm{CaCl}_{2}(5)$ & Ethanol : water $(3: 1)$ \\
\hline G25N3 & 25 & $\mathrm{NaOH}(3)$ & Ethanol \\
\hline G25N5 & 25 & $\mathrm{NaOH}(5)$ & Ethanol \\
\hline $\mathrm{G} 25 \mathrm{C} 3$ & 25 & $\mathrm{CaCl}_{2}(3)$ & Ethanol : water $(3: 1)$ \\
\hline G25C5 & 25 & $\mathrm{CaCl}_{2}(5)$ & Ethanol : water $(3: 1)$ \\
\hline G30N3 & 30 & $\mathrm{NaOH}(3)$ & Ethanol \\
\hline G30N5 & 30 & $\mathrm{NaOH}(5)$ & Ethanol \\
\hline G30C3 & 30 & $\mathrm{CaCl}_{2}(3)$ & Ethanol : water $(3: 1)$ \\
\hline G30C5 & 30 & $\mathrm{CaCl}_{2}(5)$ & Ethanol : water $(3: 1)$ \\
\hline G40N3 & 40 & $\mathrm{NaOH}(3)$ & Ethanol \\
\hline G40N5 & 40 & $\mathrm{NaOH}(5)$ & Ethanol \\
\hline G40C3 & 40 & $\mathrm{CaCl}_{2}(3)$ & Ethanol : water $(3: 1)$ \\
\hline G40C5 & 40 & $\mathrm{CaCl}_{2}(5)$ & Ethanol : water $(3: 1)$ \\
\hline
\end{tabular}

produced GO fibres in our study. In this prototype spinning line as in industrial scale devices, the main factors such as flow rate, drawing speed through methanol washing with linear velocity $1.3 \mathrm{~cm} \mathrm{~s}^{-1}$ and take up speed affecting the fibre properties were precisely controlled.

\section{Chemical reduction of GO fibres}

Three different reduction method were conducted as: (i) reduction with $\mathrm{HI}$ aqueous solution $55 \mathrm{wt} \%$, at $100{ }^{\circ} \mathrm{C},{ }^{17}$ (ii) AA reduction, $2 \mathrm{mM}$ solution in DI water at $60{ }^{\circ} \mathrm{C}^{31}$ and (iii) $\left(\mathrm{NaBH}_{4}\right)^{32}$ aqueous solution reduction. At this step, we explored the alternatives of harsh methods of hydrazine reduction and thermal reduction (see Table 1), so the proposed methodology could be easily implemented in large scale production. The reduced fibres were then washed with DI water for chemical removal and dried overnight at $60{ }^{\circ} \mathrm{C}$. A series of optimization studies was conducted based on the electrical conductivity of reduced fibres, by tuning time, temperature and concentration.

\section{Characterization of GO nanosheets, GOLCs, GO fibres and graphene fibres}

X-ray Diffraction (XRD) measurements of GO nanosheets, GO fibres and graphene fibres were performed with Bruker D-8 Advance X-Ray Diffractometer. The wavelength of irradiation of $\mathrm{Cu} \mathrm{K}$ was $0.154 \mathrm{~nm}$. The scan rate was $2.4^{\circ} \mathrm{min}^{-1}$ with the operating voltage of $40 \mathrm{kV}$ and current of $40 \mathrm{~mA}$. XRD measurements were carried out at $2 \theta$ angle ranging from $5^{\circ}$ to $90^{\circ}$. Raman spectroscopy investigations were conducted via Renishaw Raman spectrometer with a laser, excitation line at $532 \mathrm{~nm}$, at spectral range of $100-3200 \mathrm{~cm}^{-1}$. Polarized optical microscopy (POM) measurements of the GOLCs were conducted 
with a Carl Zeiss Axio Scope A1 MAT model POM in crosspolarized mode. For POM sample preparation, liquid nitrogen immersion for a few minutes in order to induce the GO layer packing for high quality imaging. To examine anisotropy of GOLCs, four different GOLCs concentrations as 20, 25, 30, $40 \mathrm{mg} \mathrm{mL}^{-1}$ were studied. Scanning electron microscope (SEM) images were performed on a field emission scanning electron microanalyzer (Zeiss LEO Supra $35 \mathrm{VP}$ ) at an acceleration voltage $5 \mathrm{kV}$ in order to monitor the orientation and packing degree of GO fibres. Mechanical properties of the GO fibres were tested by Universal Testing Machine (UTM), Shimadzu AG-X plus with $1 \mathrm{kN}$ load cell. The strain rate was $100 \mu \mathrm{m} \mathrm{min} \mathrm{m}^{-1}$ and testing speed was $0.5 \mathrm{~mm} \mathrm{~min}^{-1}$. ASTM D3379-75 standard was followed. Test specimens were prepared, as shown in Fig. S1, $\dagger$ where the as-spun GO and graphene fibres were placed into frames with fixed $3 \mathrm{~cm}$ gauge length. At least 8 specimens were tested for each material set.

Electrical conductivity was conducted on the graphene fibres with a CR-Cascade Microtech CP 4-point probe conductivity measurement device. The probe distance was $1 \mathrm{~mm}$. The electrical conductivity $(\sigma)$ was calculated from the eqn $(1)^{33}$ as:

$$
\rho=\frac{\left(\frac{\pi t}{\ln 2}\right) V}{i}=\frac{1}{\sigma}
$$
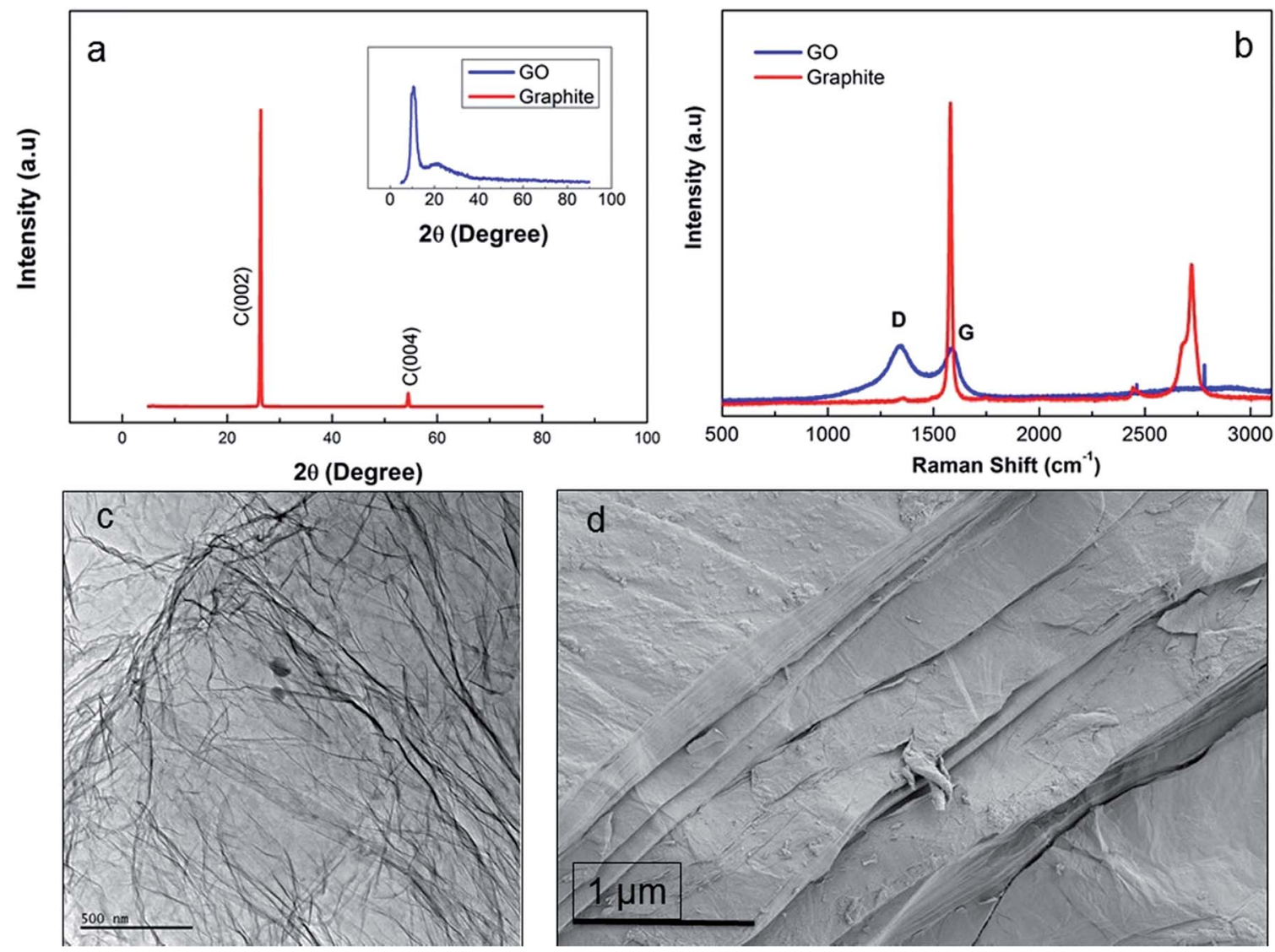

Fig. 2 (a) XRD analysis of graphite revealing (002) and (004) $h k l$ planes and as inset image GO showing $2 \theta$ peaks of hydroxyl and carboxylic pendant groups at around $9^{\circ}$ and graphene nanosheets at $23^{\circ}$, (b) Raman spectra of graphite $\left(\mathrm{G}\right.$ and $2 \mathrm{D}$ peaks at 1550 and $2750 \mathrm{~cm}^{-1}$ ) and $\mathrm{GO}(\mathrm{D}$ peak at $1370 \mathrm{~cm}^{-1}$ and $G$ peak at $1550 \mathrm{~cm}^{-1}$ ), (c) TEM and (d) SEM micrographs of GO nanosheets. where $\rho, t, V$, and $i$ are the resistivity, thickness, voltage, and current, respectively.

\section{Results and discussion}

\section{Structural analysis of GO nanosheets and characterization of GOLCs}

Fig. 2a shows the XRD pattern of synthesized functionality rich GO nanosheets revealing two different $2 \theta$ peaks of GO regarded as the proof for the presence of pendant groups and graphene nanosheets. The sharp $2 \theta$ peak at $23^{\circ}$ pointed out the presence of graphene crystalline structure while the formation of pendant functional groups was confirmed by the broad $2 \theta$ peak at around $9^{\circ} .{ }^{11}$ The rich functionalization of graphene layers through the chemical oxidation resulted in increased interlayer spacing of GO. ${ }^{\mathbf{1 1}}$ Nevertheless, addition of oxygen-containing groups intensified the defect density on the layered structure, thus disturbing the ordered graphite layer packing. The presence of graphene layers also augmented intensity of $2 \theta$ at $23^{\circ}$ while the packing disturbance prompted through oxidation led to emergence of the $2 \theta$ at $9^{\circ}$ peak in the GO diffraction compared to graphite layer. ${ }^{5}$ These two diffractions indicated that the oxidation of graphite layers was not only successful at edges, where the presence of functionalities at that region was the bulk but also its extent was enhanced towards the surface 
detected by the broad diffraction at around $2 \theta=9^{\circ}$. The promoted oxidation for improved graphite layer exfoliation was in line with the observations of $\mathrm{Xu}$ et $a l .{ }^{13}$ The intensity ratio of $\mathrm{D}$ and $\mathrm{G}$ peak $\left(I_{\mathrm{D}} / I_{\mathrm{G}}\right)$ was calculated as 0.8 and 1.04 by integration for graphite and GO, respectively (Fig. 2b). Compared to graphite, $I_{\mathrm{D}} / I_{\mathrm{G}}$ of GO nanosheets reveals that the density of functional defects was higher in GO due to the increase in interlayer spacing ${ }^{34}$ while graphite peak at $2750 \mathrm{~cm}^{-1}$ was completely removed from the GO spectrum. TEM micrograph of individual GO sheets (Fig. 2c) showed that large, few-layers of GO can be obtained where physical perturbations were minimized. However, SEM micrograph (Fig. 2d) revealed the multiple layer stacking in solid phase. Thus, one of the prerequisites to produce $3 \mathrm{D}$ structured GO fibres, is to have ordered GOLCs where the aligned GO nanosheets has been succeeded (Fig. 3). ${ }^{28}$

The alignment of GOLCs was evaluated in cross-polarized mode POM. The birefringence behaviour of GOLCs assisted to the detection of distinctive domains visible by different contrasts (Fig. 3), which pointed out the local alignment of GO layers in the liquid crystal phase. This local GO stacking reflected the ordinary and extraordinary rays of the polarized light by different angles, thus due to the cross-polarization of reflected light, local contrast differences were detected. To explore GOLCs concentration effect on phase formations, first GOLCs at 20 and $25 \mathrm{mg} \mathrm{mL} \mathrm{m}^{-1}$ concentrations were studied. Fig. $3 a$ and $b$ revealed the nematic phase formation at these concentrations, whereas due to relatively short-range order of GO nanosheets, the birefringence was quite limited. This effect was interpreted from the few numbers of birefringent sites with different contrasts in their respective POM images. ${ }^{10}$ At $30 \mathrm{mg} \mathrm{mL}{ }^{-1}$ GO concentration, more distinctive GOLCs alignment leading multiple birefringence was observed (Fig. 3c). The clear change in contrast and colour of nematic phase at higher concentrations was the result of their relatively different interference of ordinary and extraordinary light rays. ${ }^{35}$ By varying the incident angle and so the interference of each locally aligned stacks, the colour contrast map changed and this enabled to visualize a series of local orientation sites in a single specimen. ${ }^{9}$ Fig. 3d displayed better alignment compared to lower concentrations so it is predicted that spinning at this concentration tends to form denser and stronger fibres due to long range order of GO layer stacks. ${ }^{10,25}$

\section{Continuous GO fibre production via wet-spinning line}

The continuous GO fibres were produced through a customized spinning line, where spinning and take-up speed were controlled effectively. A syringe pump to dispense GOLCs was used to control feeding rate. The spinneret designed with $d / L>$ 150 ratio was preferred to assist further alignment towards the flow direction for higher layer packing, ultimately for better fibre properties. Then, additional washing step was added to remove the coagulation bath residues by the aid of hydroxide groups in methanol. At this step, the residual $\mathrm{NaOH}$ and $\mathrm{CaCl}_{2}$ moieties remained on fibres was also neutralized, thus improving the green nature of GO fibres. Besides, $\mathrm{H}_{2} \mathrm{O}$ molecules interacted with GO was partially removed via dehydration of fibres, promoting an easier drying process. As an experimental point of view, the results showed that unwashed fibres had entrapped $\mathrm{H}_{2} \mathrm{O}$ molecules even after three days while the methanol washed fibres were fully dry within a few minutes.
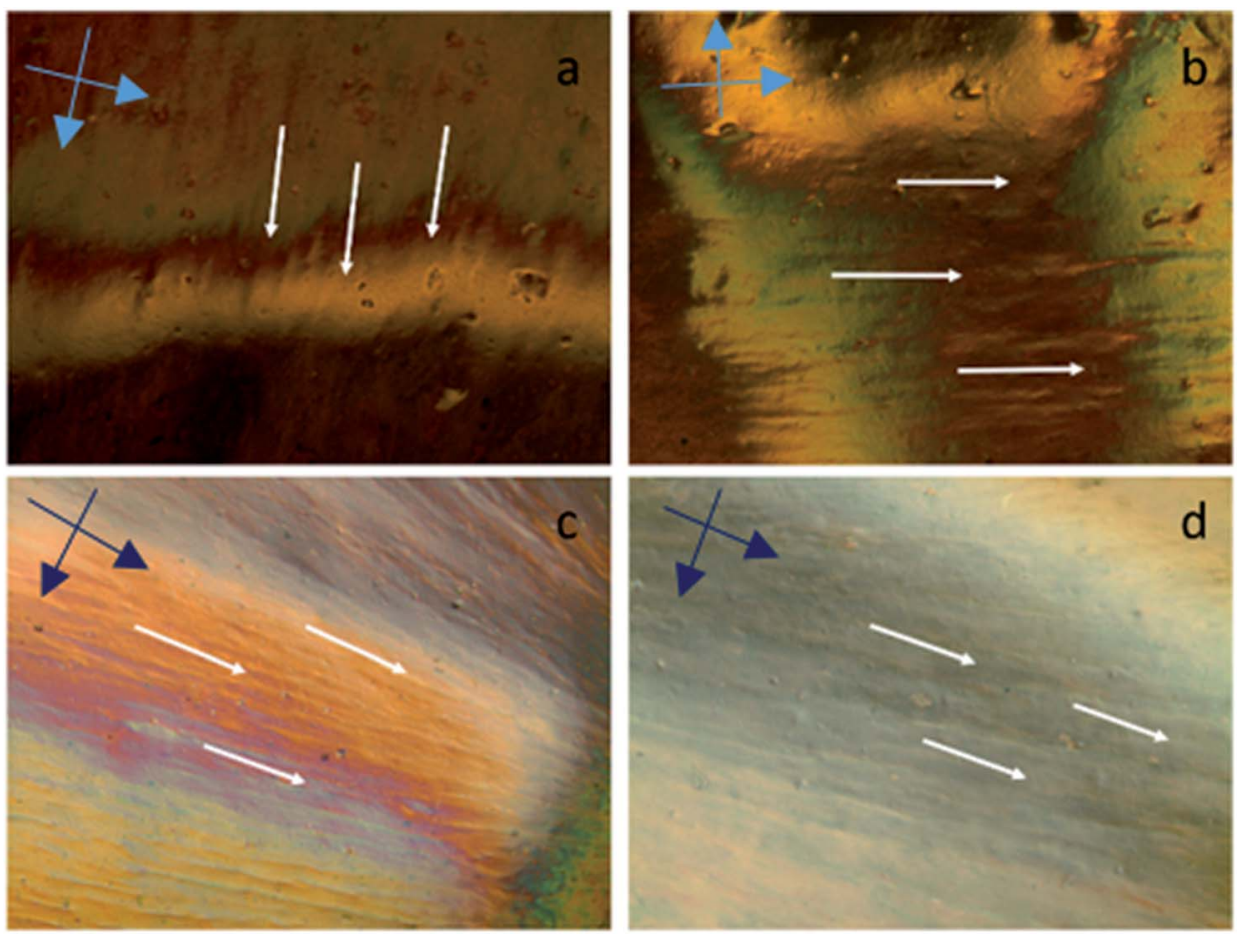

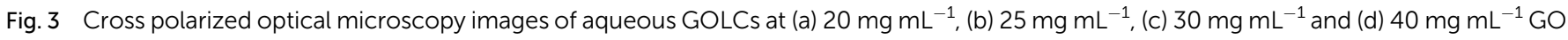
concentrations. The cross dark arrows represent the cross-polarization direction. 
Thus these dry fibres could be easily collected by a rotating drum without any noticeable damage.

The effect of coagulation media on structural and mechanical properties of fibres is still intriguing. Herein, we systematically studied the material parameters such as coagulation chemicals (e.g. $\left.\mathrm{CaCl}_{2}, \mathrm{NaOH}\right)$, their concentrations (e.g. $3 \mathrm{wt} \%, 5 \mathrm{wt} \%$ ) and coagulation composition (e.g. ethanol, ethanol : water), as detailed in Table 2. Fibres coagulated in $\mathrm{CaCl}_{2}$ coagulation bath (Fig. 4a-d) showed higher packing density compared to their equivalent GO fibres coagulated in $\mathrm{NaOH}$ (Fig. $4 \mathrm{f}$ to h). Nevertheless, we should emphasize that as in G40C5 and G40N5 fibres coagulated in $\mathrm{NaOH}$ (Fig. $4 \mathrm{~d}$ and $\mathrm{h}$ ), the packing density could be enhanced by increasing GO content. At such high GOLCs concentrations, the infusion of metal ions in coagulation bath could be prevented due to GO layer alignment and packing. ${ }^{28}$ Thus, Fig. $4 \mathrm{~d}$ and h exhibited similar packing densities free from coagulation medium. As expected, the surface topologies of low GO concentration fibres (e.g. $20 \mathrm{mg} \mathrm{mL}^{-1}$ and $25 \mathrm{mg} \mathrm{mL}^{-1}$ ) were significantly different from high GO concentration fibres (e.g. $30 \mathrm{mg} \mathrm{mL}^{-1}$ and $40 \mathrm{mg} \mathrm{mL}^{-1}$ ) in both coagulation media. The GO fibres produced from $20 \mathrm{mg} \mathrm{mL}^{-1}$ (Fig. $4 \mathrm{a}$ and e), and $25 \mathrm{mg}$ $\mathrm{mL}^{-1}$ suspensions (Fig. $4 \mathrm{~b}$ and $\mathrm{f}$ ) had noticeable wrinkling effect and some irregular features. When GO concentration increased to $30 \mathrm{mg} \mathrm{mL}^{-1}$ (Fig. $4 \mathrm{c}$ and g) and $40 \mathrm{mg} \mathrm{mL}^{-1}$ (Fig. $4 \mathrm{~d}$ and $\mathrm{h}$ ), such loose and irregular features were transformed to highly packed and extremely aligned structures. These densely packed fibres could withstand drawing and further finishing stresses during spinning, with showing higher tensile strength. Therefore, we observed that fibre formation was more successful even up to several meters at higher GOLCs concentration (e.g. $30 \mathrm{mg}$ $\mathrm{mL}^{-1}$ and $40 \mathrm{mg} \mathrm{mL}^{-1}$ ) (Fig. 1). It is noteworthy that the reservoir capacity significantly affects the final fibre length in our spinning line and as predicted, larger reservoir leads to longer fibres. ${ }^{\mathbf{1 3 2 9}}$

\section{Mechanical properties of GO fibres}

GOLCs concentration, coagulation bath media and stress applied during spinning process has major effect on the final fibre properties. First, tensile properties of $40 \mathrm{mg} \mathrm{mL}{ }^{-1} \mathrm{GO}$ fibres (Fig. 5a) and $30 \mathrm{mg} \mathrm{mL} \mathrm{m}^{-1} \mathrm{GO}$ fibres (Fig. 5b) coagulated in different media (detailed in Table 2) were compared. Each single fibre was implemented into UTM as shown in Fig. 5c, where the cross-section of fibres might be varied due to their crumbled layered structures. Thus, as displayed in Table 3, specific ultimate strength (N/tex) was also calculated for each fibre class to eliminate fibre diameter effect due to the different ion diffusion mechanism in $\mathrm{CaCl}_{2}$ and $\mathrm{NaOH}$ media. The results suggested that enhancement in the ultimate tensile strength of as-spun GO fibres due to higher GO concentration and GO stacking had minor effect about $10 \%$ depending on the coagulation medium, whereas strain to failure (\%) decreased in general.

Fig. 5a pointed out that at same GO concentration level, the preference of $\mathrm{CaCl}_{2}$ coagulation bath resulted in higher ultimate
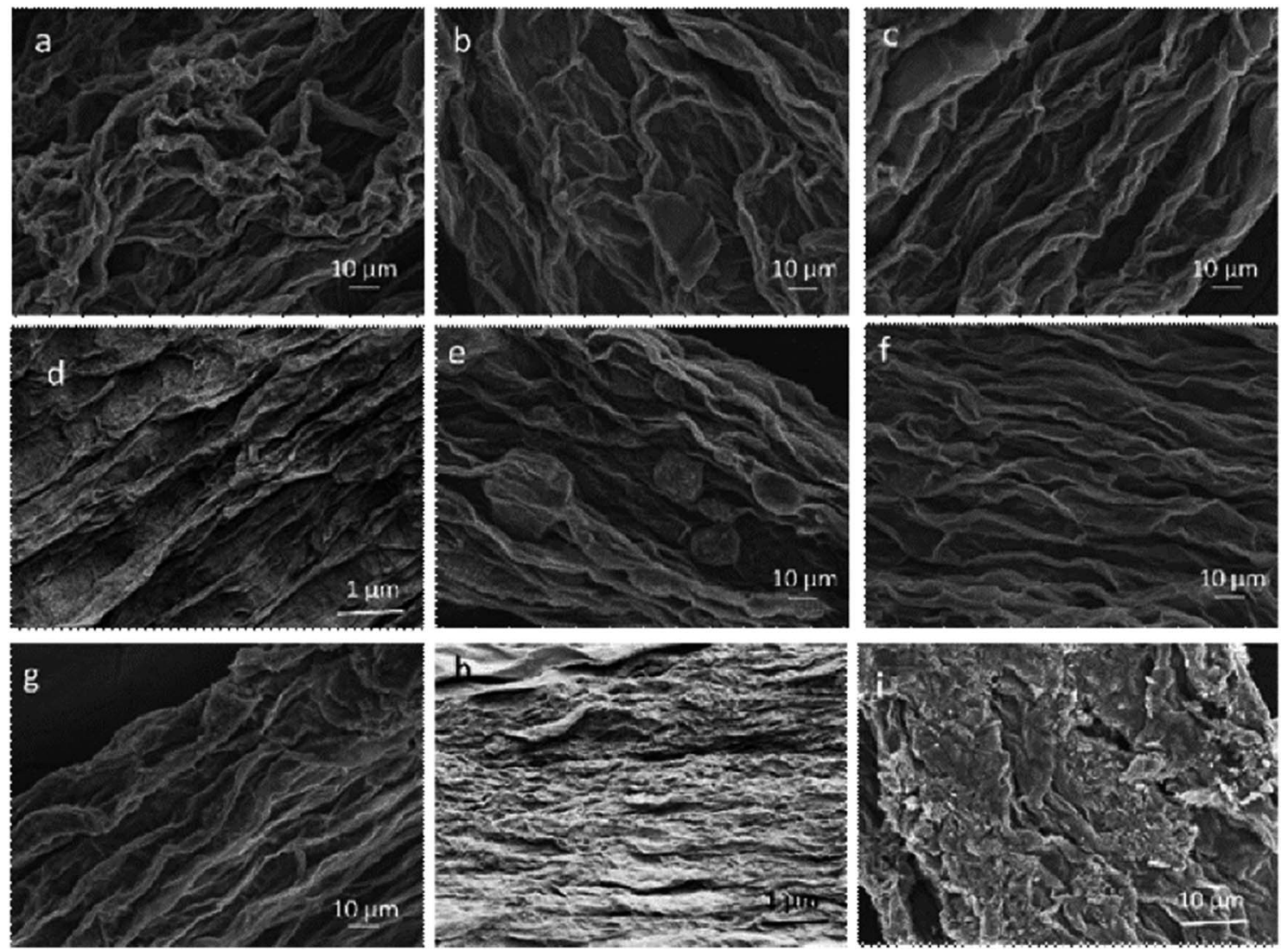

Fig. 4 SEM images of as-spun (a) G20C5, (b) G25C5, (c) G30C5, (d) G40C5, (e) G20N5, (f) G25N5, (g) G30N5, (h) G40N5 coded GO fibres taken from their surfaces and (i) fracture surface of as-spun G30C5 fibre after tensile testing. 

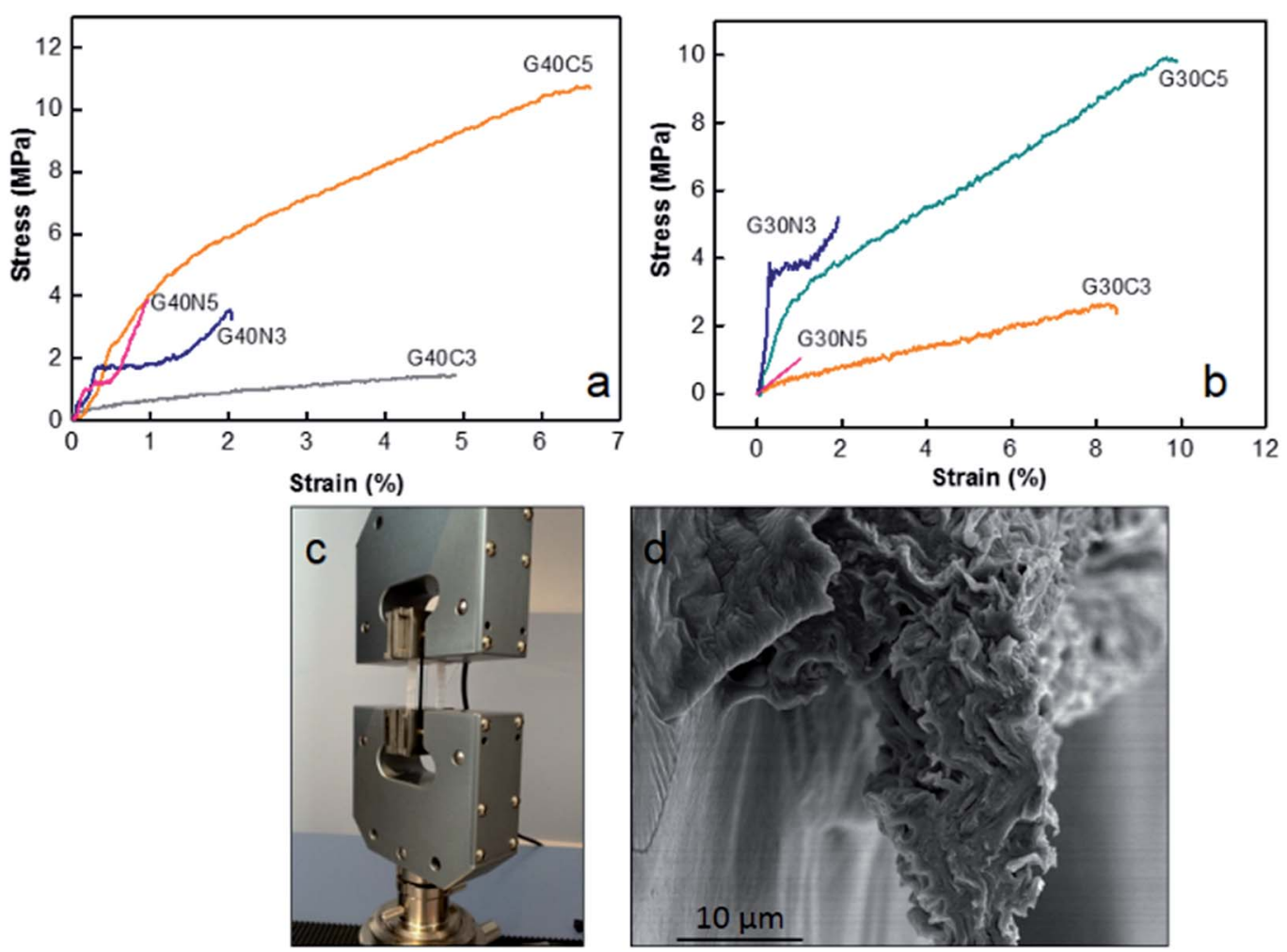

Fig. 5 (a) Stress $(\sigma)$-strain $(\varepsilon)$ curve of $40 \mathrm{mg} \mathrm{mL}^{-1} \mathrm{GO}$ coded fibres, (b) stress $(\sigma)$-strain $(\varepsilon)$ curve of $30 \mathrm{mg} \mathrm{mL}^{-1}$ as-spun GO coded fibres, (c) implementation of single GO fibre for tensile testing (d) SEM image of the fracture surface of as-spun G40C5 fibre after testing revealing intrinsic crumbled structure.

Table 3 Tensile properties of GO fibres coded in Table 2 and reference studies from literature

\begin{tabular}{llcc}
\hline GO fibre & $\begin{array}{l}\text { Diameter } \\
(\mu \mathrm{m})\end{array}$ & $\begin{array}{l}\text { Strain to } \\
\text { failure }(\%)\end{array}$ & $\begin{array}{l}\text { Specific ultimate } \\
\text { strength (N/tex) }\end{array}$ \\
\hline As-spun G30C5 & 130 & $10 \pm 2.1$ & $7 \pm 0.5$ \\
As-spun G40C5 & 130 & $6.7 \pm 0.5$ & $5.9 \pm 1.3$ \\
Reduced G30C5 & 229 & $4.7 \pm 0.5$ & $4.5 \pm 2$ \\
Reduced G40C5 & 271 & $2.8 \pm 1.7$ & $14.5 \pm 0.4$ \\
As-spun G30N5 & 189 & $1.3 \pm 0.2$ & $5.8 \pm 1.5$ \\
As-spun G40N5 & 203 & $0.9 \pm 0.1$ & $6.4 \pm 0.7$ \\
GO fibre ref. 26 & 26 & $1.64 \pm 0.12$ & $1.04 \pm 0.01$ \\
(draw ratio 1.09) & & & \\
GO fibre ref. 26 & 26 & $0.84 \pm 0.08$ & $1.26 \pm 0.01$ \\
(draw ratio 1.27) & & & \\
Reduced GO fibre & 240 & $1.5 \pm 0.1$ & $1.02 \pm 0.03$ \\
ref. 38 & & & \\
\end{tabular}

strength and strain to failure (\%). For instance, G40C5 fibres coagulated in $5 \mathrm{wt} \% \mathrm{CaCl}_{2}$ medium exhibited 2 fold higher ultimate strength and 5-fold increase in strain to failure compared to as-spun G40N5. Even though the same amount of GO dispensed, as shown in Table 3, mean fibre diameters differed drastically due to differences in ion diffusion mechanism. It is clear that $\mathrm{CaCl}_{2}$ coagulation media which contains divalent $\mathrm{Ca}^{2+}$ ions, contributed to the fibre toughening and produced compact thinner fibres. The interaction between divalent metal ions $\left(\mathrm{Ca}^{2+}\right)$ and the carboxylic groups of the GO nanosheets has been explained by Park et al. ${ }^{36}$ They stated that divalent ions easily diffused into the fibre's interior, thus reacted completely with functional sites on the edges of GO sheets, which improved the toughness of the fibres. ${ }^{36}$ Our results showed that not only ultimate strength but also strain to failure \% was affected from these interactions. The affinity of $\mathrm{Ca}^{2+}$ ions towards GO sheets caused compact crumbled intrinsic morphologies and finally finer fibres. Higher number density of contact sites between each nanosheet induced much higher ultimate strain \% and assisted plastic deformation mechanism. Meanwhile, the coagulation in $\mathrm{NaOH}$ media promoted in situ partial reduction of GO layers, thus reducing the possibility of hydrogen bond network formation among the functional groups at the GO basal planes. ${ }^{\mathbf{1 2 , 2 2}}$ It is noteworthy that GO concentration has minor effect on tensile properties ${ }^{11,37}$ while packing density and hydrogen bond network ${ }^{26}$ promoted by coagulation process significantly dominate the mechanical response of fibres. Fig. $4 \mathrm{i}$ and 5d demonstrated the fracture surfaces of G30C5 and G40C5, respectively, where G40C5 fibres showed lower ductility than G30C5 fibres. Higher GO content per specific area contributed to brittleness of fibres due to the restrictions on plasticization. Restricted void formation and stress concentration sites might increase the strength while trading the ductility off. Overall, our results have shown that increasing GO content and packing density may alter fibre strength which would result in further changes in segmental mobility and depression of strain to failure (\%). 

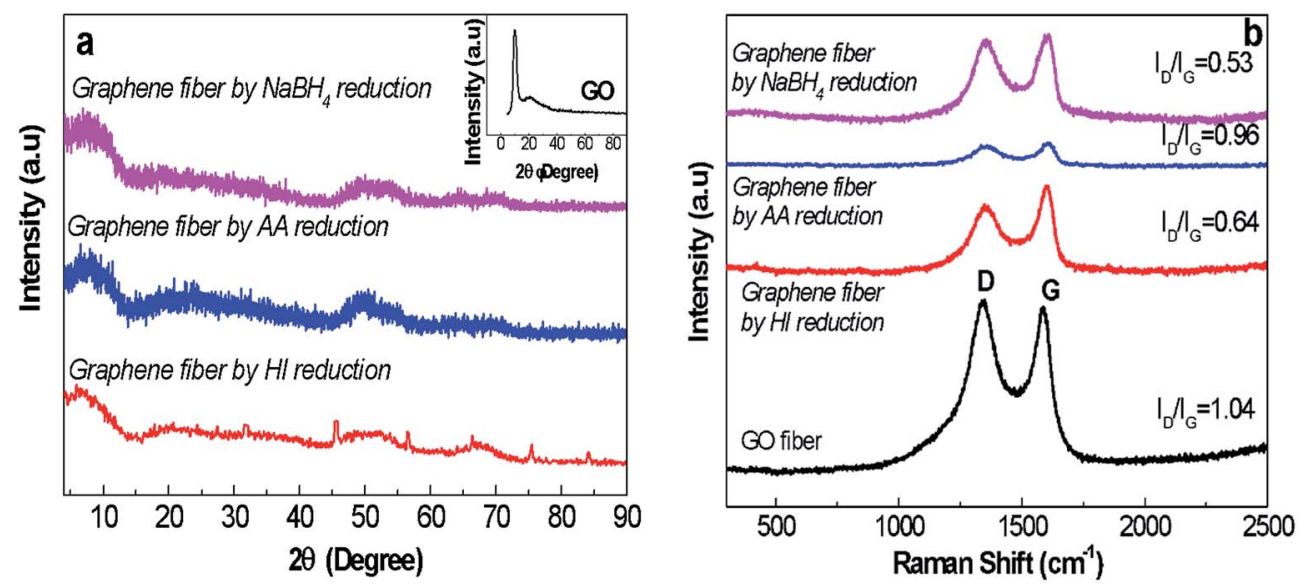

Fig. 6 (a) XRD spectra (the inset figure represents the XRD spectrum of GO nanosheets as detailed in Fig. 2) and (b) Raman spectra of GO and graphene fibres reduced by $\mathrm{NaBH}_{4}, \mathrm{AA}$ and $\mathrm{HI}$ agents revealing $\mathrm{D}$ peak at $1370 \mathrm{~cm}^{-1}$ and $\mathrm{G}$ peak at $1550 \mathrm{~cm}^{-1}$.

As summarized in Table 3, compared to reference studies, proposed coagulation and washing protocol assembled more flexible (about 9-fold) continuous GO fibres without impeding stiffness. ${ }^{26,38}$

\section{Continuous graphene fibres via chemical reduction}

Structural analysis of graphene fibres. The lack of electron conduction mechanism of as-spun GO fibres definitely limits the use of these fibres in sensing and electronic applications. To gain electrical conductivity, among several approaches chemical reduction was preferred due to possessing lower pollution and energy consumption. ${ }^{31,32}$ Besides, instead of using harsh reduction methods such as hydrazine and thermal treatment, we explored an efficient green strategy to provide electrical conductivity. Thus, reduction process parameters including reaction time and temperature were also studied. Meanwhile these methods provide higher flexibility and versatility, and most importantly cause the reduction of graphene sheet disturbance (e.g. breakage and unintended layer dislocation) without sacrifying mechanical performance of fibres. ${ }^{39}$ Herein, flexible G30C5 fibres with the highest specific strength and larger ductility were selected for further chemical reduction studies. Fig. 6a shows the suppression of functional groups presented in GO due to $\mathrm{NaBH}_{4}$, AA and HI reduction, interpreted as decreased $2 \theta$ at $9^{\circ}$ in XRD spectra. Distinctively, $\mathrm{NaBH}_{4}$ reduction process, enabled the highest reduction degree in terms of interlayer spacing. Raman characterization of graphite and GO (Fig. 6b) pointed out that the D peak at 1350 $\mathrm{cm}^{-1}$ was detected only for GO structure while the G peak, which indicates $E_{2 \mathrm{~g}}$ mode of graphite layers, was seen at 1600 and $1650 \mathrm{~cm}^{-1}$ for graphite and GO, respectively. ${ }^{27}$ The disruption of interlayer spacing leaded to $\mathrm{G}$ peak shifts in GO compared to graphite. ${ }^{40}$ The D peak of Raman spectra of the graphene-based materials emerged in high intensity due to formation of structural defects within their layered formation of nanosheets. ${ }^{41}$ The comparison between the $I_{\mathrm{D}} / I_{\mathrm{G}}$ ratio of asspun GO and reduced graphene fibres showed the level of success in elimination of functional group-driven defects, interpreted from reduced $I_{\mathrm{D}} / I_{\mathrm{G}}$ ratio. Fig. $6 \mathrm{~b}$ suggests that $\mathrm{NaBH}_{4}$ reduction approach $\left(I_{\mathrm{D}} / I_{\mathrm{G}}=0.53\right)$ provided the most successful route to remove the structural defects.

Electrical conductivity of graphene fibres. We first attempt to reveal the effects of reducing agent and their relative reaction times on electrical conductivity of reduced fibres. So far it is assumed that 24 hours of reduction time would be sufficient to reach stability for electrical conductivity. ${ }^{22}$ However, Fig. 7a unveiled that the highest electrical conductivity of $73 \mathrm{~S} \mathrm{~cm}^{-1}$ was achieved by $\mathrm{NaBH}_{4}$ reduction after 72 hours. Hence, $\mathrm{NaBH}_{4}$ reduction method was selected for further studies to explore temperature and concentration effect. The effect of temperature on electrical conductivity of $\mathrm{NaBH}_{4}$ reduced fibres (Fig. 7b) was investigated. Reduction kinetics at $90{ }^{\circ} \mathrm{C}$ yielded 3 fold higher electrical conductivity compared to reduction at 30 and $60{ }^{\circ} \mathrm{C}$. Overall, $\mathrm{NaBH}_{4}$ reduction at $90{ }^{\circ} \mathrm{C}$ for 72 hours reached highest conductivity of $255 \mathrm{~S} \mathrm{~cm}^{-1}$. Graphene fibres exhibited semiconductor characteristics as displayed in Fig. 7b, as temperature increases, conductance increases resulting in dwindling of resistance. It could be associated to excitation of valence band electrons by temperature, as observed in semiconductors.

In shorten reaction times at $90{ }^{\circ} \mathrm{C}$, we enquired into $\mathrm{NaBH}_{4}$ concentration effect (from $0.3 \mathrm{M}$ to $2.4 \mathrm{M}$ ). Fig. 7c revealed the highest electrical conductivity of $214 \mathrm{~S} \mathrm{~cm}^{-1}$ along fibre axis prevailed at concentration of $2.4 \mathrm{M} \mathrm{NaBH}_{4}$. The high conductivity of graphene fibres is believed to be due to increased number of reduced sites. This effect further leads to higher conductivity of reduced graphene fibres while preserving the oriented fibre structure which minimized the contact resistance. The combination of good flexibility, high tensile strength and enhanced electrical conductivities of graphene fibres make stretchable wires for promising wearable functional textiles. Thus, G30C5 and G40C5 as-spun GO fibres reduced by eco-friendly $\mathrm{NaBH}_{4}(2.4 \mathrm{M})$ at $90{ }^{\circ} \mathrm{C}$ for 24 hours were tested under tensile loading, in next section.

Mechanical properties of graphene fibres. To calculate the mechanical behaviour of graphene fibres and to monitor the effect of reduction process, we performed tensile testing of both reduced G30C5 and G40C5 fibres. The mechanical tests described in 

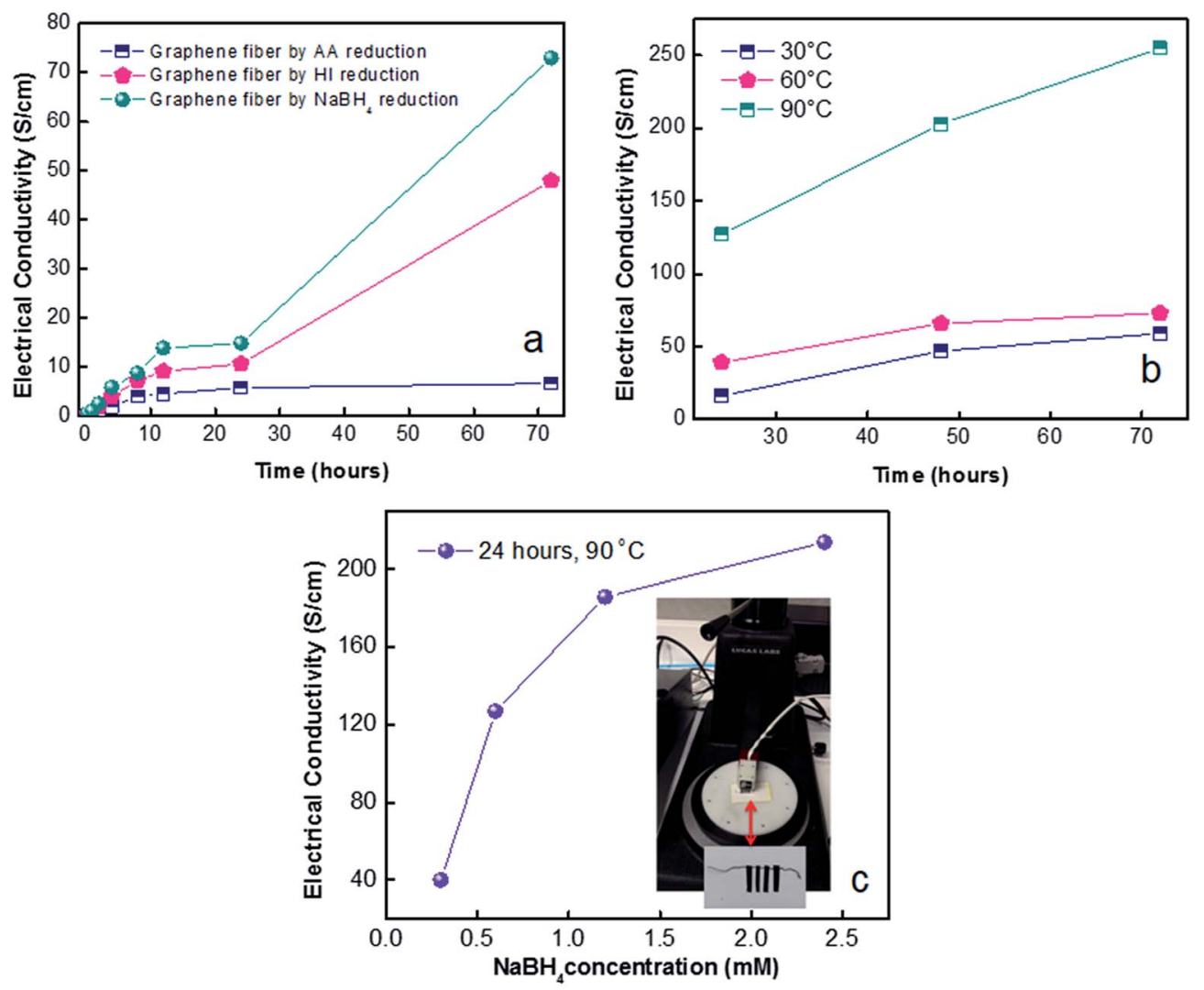

Fig. 7 Electrical conductivity of graphene fibres by revealing (a) effect of reducing agent depending on reaction time, (b) effect of reduction times depending on temperature for $\mathrm{NaBH}_{4}(1.2 \mathrm{M})$ reducing agent, (c) effect of $\mathrm{NaBH}_{4}$ concentration.

Experimental section were carried out to determine the stressstrain behaviour along fibre direction (Fig. 8). Table 3 also summarizes ultimate tensile strength and strain to failure (\%) of G30C5 and G40C5 graphene fibres. Here, we first investigate the change in strain to failure (\%) during the process of reduction. $\mathrm{NaBH}_{4}$ reduction caused swollen fibres, which failed in brittle manner. This change in ductility could be associated to prevented slipping of graphene layers due to the corrupted network after reduction. Furthermore, depending on GO density, ultimate specific strength might be increased or decreased.$^{27}$ For instance,

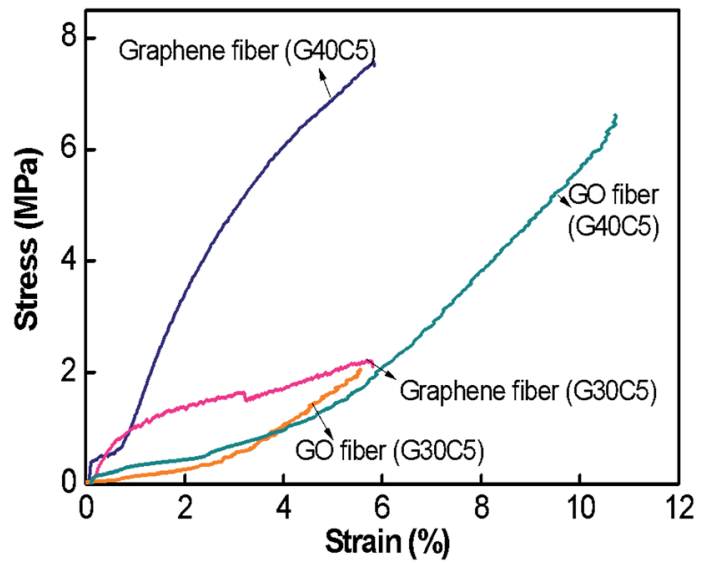

Fig. 8 Stress $(\sigma)$-strain $(\varepsilon)$ curves of G30C5, G40C5 GO fibres and reduced $\mathrm{G} 30 \mathrm{C} 5$ and $\mathrm{G} 40 \mathrm{C} 5$ graphene fibres. tensile ultimate strength drastically improved for reduced G40C5 graphene fibres, whereas there is slight reduction observed in reduced G30C5 graphene fibres. Apart from the spinning process, the structural difference of these fibres came from the presence of higher number of reduction sites. The graphene fibres with higher degree of reduction as in reduced G40C5 fibres were less affected from this post process in terms of tensile strength whereas high number density of stiff graphene nanosheets prevented plastic deformation and caused brittle failure. We should also note that the specific stress of reduced G30C5 graphene fibres was higher compared previous studies as described in Table 3.

\section{Conclusion}

Scalable production of graphene oxide and graphene fibres from stable aqueous GOLCs were fabricated via the designed continuous wet-spinning line while process (e.g. the feeding rate, take up speed and drawing speed) and material parameters including coagulation composition and concentration were precisely controlled through the proposed green approach. The results show that as-spun $\mathrm{GO}$ fibres coagulated in $\mathrm{CaCl}_{2}$ ensured promising tensile properties and GO fibres showed strain to failure (\%) of $10 \%$ with higher specific ultimate strength. $\mathrm{NaBH}_{4}$ reduction approach $\left(I_{\mathrm{D}} / I_{\mathrm{G}}=0.53\right)$ performed at $90{ }^{\circ} \mathrm{C}$ for 24 hours with $2.4 \mathrm{M}$ concentration, enabled facile route to gain electrical conductivity while individual reduced G30C5 fibres exhibited electrical conductivity of $214 \mathrm{~S} \mathrm{~cm}^{-1}$ 
along fibre axis. Overall we suggest that green and scalable production of graphene fibres could have promising applications in sensing and wearable electronics since they are nontoxic, flexible and have high tensile strength.

\section{Acknowledgements}

This research has received funding from the Scientific and Technological Research Council of Turkey (TUBITAK) under project number 214M398. The authors thank to MSc student Ismail Kaya for his help in wet spinning set up.

\section{References}

1 L. Işıkel Şanlı, V. Bayram, B. Yarar, S. Ghobadi and S. Alkan Gürsel, Int. J. Hydrogen Energy, 2016, 41, 3414-3427.

2 F. Lin, X. Tong, Y. Wang, J. Bao and Z. M. Wang, Nanoscale Res. Lett., 2015, 10, 435.

3 L. Işıkel Şanlı, V. Bayram, S. Ghobadi, N. Düzen and S. Alkan Gürsel, Int. J. Hydrogen Energy, 2017, 42, 1085-1092.

4 H.-P. Cong, J.-F. Chen and S.-H. Yu, Chem. Soc. Rev., 2014, 43, 7239-7456.

5 S. Ghobadi, S. Sadighikia, M. Papila, F. Ç. Cebeci and S. A. Gürsel, RSC Adv., 2015, 5, 85009-85018.

6 S. Ghobadi, S. Mehraeen, R. Bakhtiari, B. Shamloo, V. Sadhu, M. Papila, F. Ç. Cebeci and S. A. Gürsel, RSC Adv., 2016, 6, 92434-92442.

7 J. Li, G. Wang, H. Zhu, M. Zhang, X. Zheng, Z. Di, X. Liu and X. Wang, Sci. Rep., 2014, 4, 4359.

8 T. Mosciatti, S. Haar, F. Liscio, A. Ciesielski, E. Orgiu and P. Samori, ACS Nano, 2015, 9, 2357-2367.

9 Z. Xu and C. Gao, Acc. Chem. Res., 2014, 47, 1267-1276.

10 R. Jalili, S. H. Aboutalebi, D. Esrafilzadeh, R. L. Shepherd, J. Chen, S. Aminorroaya-Yamini, K. Konstantinov, A. I. Minett, J. M. Razal and G. G. Wallace, Adv. Funct. Mater., 2013, 23, 5345-5354.

11 Z. Xu and C. Gao, Nat. Commun., 2011, 2, 571.

12 P. Kumar, U. N. Maiti, K. E. Lee and S. O. Kim, Carbon, 2014, 80, 453-661.

13 Z. Xu, H. Sun, X. Zhao and C. Gao, Adv. Mater., 2013, 25, 188193.

14 Z. Liu, Z. Li, Z. Xu, Z. Xia, X. Hu, L. Kou, L. Peng, Y. Wei and C. Gao, Chem. Mater., 2014, 26, 6786-6795.

15 X. Yang, C. Guo, L. Ji, Y. Li and Y. Tu, Langmuir, 2013, 29, 8103-8107.

16 K. E. Lee, J. J. Oh, T. Yun and S. O. Kim, J. Solid State Chem., 2015, 224, 115-119.

17 Z. Tian, C. Xu, J. Li, G. Zhu, Z. Shi and Y. Lin, ACS Appl. Mater. Interfaces, 2013, 5, 1489-1493.

18 B. Dan, N. Behabtu, A. Martinez, J. S. Evans, D. V. Kosynkin, J. M. Tour, M. Pasquali and I. I. Smalyukh, Soft Matter, 2011, 7, 11154-11159.

19 N. Behabtu, J. R. Lomeda, M. J. Green, A. Sinitskii, D. V. Kosynkin, J. Schmidt, E. Kesselman, M. Pasquali, A. L. Higginbotham, D. Tsentalovich, Y. Talmon,
A. N. G. Parra-Vasquez, J. M. Tour and Y. Cohen, Nat. Nanotechnol., 2010, 5, 406-411.

20 A. H. Wazir and L. Kakakhel, New Carbon Mater., 2009, 24, 83-88.

21 H. P. Cong, X. C. Ren, P. Wang and S. H. Yu, Sci. Rep., 2012, 2, 613.

22 L. Chen, Y. He, S. Chai, H. Qiang, F. Chen and Q. Fu, Nanoscale, 2013, 5, 5809-5815.

23 W. Chen, L. Yan and P. R. Bangal, Carbon, 2010, 48, 11461152.

24 D. C. Marcano, D. V. Kosynkin, J. M. Berlin, A. Sinitskii, Z. Sun, A. Slesarev, L. B. Alemany, W. Lu and J. M. Tour, ACS Nano, 2010, 4, 4806-4814.

25 G. Huang, C. Hou, Y. Shao, H. Wang, Q. Zhang, Y. Li and M. Zhu, Sci. Rep., 2014, 4, 4248.

26 C. Xiang, C. C. Young, X. Wang, Z. Yan, C. C. Hwang, G. Cerioti, J. Lin, J. Kono, M. Pasquali and J. M. Tour, $A d v$. Mater., 2013, 25, 4592-4597.

27 S. Hussain, C. Yorucu, I. Ahmed, R. Hussain, B. Chen, M. Bilal Khan, N. A. Siddique and I. U. Rehman, Surf. Coat. Technol., 2014, 258, 458-466.

28 Z. Dong, C. Jiang, H. Cheng, Y. Zhao, G. Shi, L. Jiang and L. Qu, Adv. Mater., 2012, 24, 1856-1861.

29 S. Zhang, K. K. Koziol, I. A. Kinloch and A. H. Windle, Small, 2008, 4, 1217-1222.

30 J. W. S. Hummers and R. E. Offeman, J. Am. Chem. Soc., 1958, 80, 1339.

31 M. J. Fernández-Merino, J. I. P. L. Guardia, S. Villar-Rodil, P. Solís-Fernández, A. Martínez-Alonso and J. M. D. Tascón, J. Phys. Chem. C, 2010, 114, 6426-6432.

32 H.-J. Shin, K. K. Kim, A. Benayad, S.-M. Yoon, H. K. Park, I.-S. Jung, M. H. Jin, H.-K. Jeong, J. M. Kim, J.-Y. Choi and Y. H. Lee, Adv. Funct. Mater., 2009, 19, 1987-1992.

33 D. S. McLachlan, J. Phys. C: Solid State Phys., 1985, 18, 18911897.

34 F. T. Thema, M. J. Moloto, E. D. Dikio, N. N. Nyangiwe, L. Kotsedi, M. Maaza and M. Khenfouch, J. Chem., 2013, 2013, 6 .

35 R. Niu, J. Gong, D. Xu, T. Tang and Z.-Y. Sun, Colloids Surf., A, 2015, 470, 22-30.

36 S. Park, K.-S. Lee, G. Bozoklu, W. Cai, S. T. Nguyen and R. S. Ruoff, ACS Nano, 2008, 2, 572-578.

37 Z. Dong, C. Jiang, H. Cheng, Y. Zhao, G. Shi, L. Jiang and L. Qu, Adv. Mater., 2012, 24, 1856-1861.

38 S. Chen, W. Ma, Y. Cheng, Z. Weng, B. Sun, L. Wang, W. Chen, F. Li, M. Zhu and H. M. Cheng, Nano Energy, $2015,15,642-653$.

39 S. Pei, J. Zhao, J. Du, W. Ren and H.-M. Cheng, Carbon, 2010, 48, 4466-4474.

40 L. I. Șanlı, V. Bayram, B. Yarar, S. Ghobadi and S. A. Gürsel, Int. J. Hydrogen Energy, 2016, 41, 3414-3427.

41 S. Stankovich, D. A. Dikin, R. D. Piner, K. A. Kohlhaas, A. Kleinhammes, Y. Jia, Y. Wu, S. T. Nguyen and R. S. Ruoff, Carbon, 2007, 45, 1558-1565. 